\title{
Electric Field Enhancement Control in Active Junction of IGBT Power Module
}

\author{
Abdelghaffar Amoka Abdelmalik ${ }^{1^{*}}$ and Knut Brede Liland ${ }^{2}$ \\ ${ }^{1}$ Department of Physics, Ahmadu Bello University Zaria, 810002, Nigeria \\ ${ }^{2}$ SINTEF Energy Research, Sem Sælands vei 11, 7034, Trondheim, Norway \\ *Corresponding author: aaabdelmalik@abu.edu.ng
}

Published online: 25 November 2020

To cite this article: Abdelmalik, A. A. \& Liland, K. B. (2020). Electric field enhancement control in active junction of IGBT power module. J. Phys. Sci., 31(3), 1-15. https://doi.org/10.21315/jps2020.31.3.1

To link to this article: https://doi.org/10.21315/jps2020.31.3.1

\begin{abstract}
Dielectric liquids are incompressible, able to fill voids and have selfhealing effect and hence are being considered as alternative encapsulation materials to establish power electronics at ambient high pressure at subsea conditions. In a long-term endurance test, insulated-gate bipolar transistor (IGBT) chips subjected to $6.5 \mathrm{kV} \mathrm{DC}$ stress in dielectric oil environment was reported to have failed after less than one week in operation. A critical look at the failed objects revealed contamination fibres at the surface and around the high field regions. This paper presents the numerical simulation of field distribution around a conducting fibre at the surface of the IGBT chip. It also evaluates the influence of the nature of the encapsulation material on the integrity of power electronic modules using a long-term experiment at a medium elevated temperature for high and low relative humidity operated close to service load using IGBT relevant chips. Finite element method (FEM) calculations show how the high field region can be shielded from impurities that can easily trigger partial discharge (PD) and breakdown. The simulation suggests that coating the surface of the module with a thin polymer layer with a thickness of $20 \mu \mathrm{m}$ or more could be sufficient to improve the reliability of the encapsulation system. Additional polymer coat with thickness $27 \mu \mathrm{m}$ on the chip made the system survive without failure for 67 weeks under test and dry operating condition. Meanwhile, thick coating such as silicone gel protected the object longer under higher relative humidity.
\end{abstract}

Keywords: Dielectric breakdown, electric field, finite element methods, insulated gate bipolar transistor module, insulation 


\section{INTRODUCTION}

Development trends in power electronic systems are moving towards higher voltages and new applications such as subsea pressurised converters. This calls for the development of new insulation systems. Today, silicone gels are dominating as a coating material while compressible sulphur hexafluoride $\left(\mathrm{SF}_{6}\right)$ is used in presspack modules. Incompressible silicone gels as coating could be replaced by other materials. Dielectric liquids being incompressible and able to fill voids are considered as an alternative to establishing power electronics at ambient high pressure at subsea conditions.

This brings about the introduction of a new generation of $1.7 \mathrm{kV}, 3.3 \mathrm{kV}, 4.5 \mathrm{kV}$ and $6.5 \mathrm{kV}$ range of high voltage (HV) insulated gate bipolar transistor (IGBT) modules as part of the active components of the power converter. The recently introduced $6.5 \mathrm{kV}$ HV IGBT modules were designed to substantially reduce the total semiconductor loss while increasing the turn-off ruggedness. ${ }^{1,2}$ The increase in voltage switching demand for a better insulation capability and the module's resistance to partial discharges (PDs) in the high field region.

A cross-section model of the power electronic module shown in the lower part of Figure 1 consists of aluminium nitride (AlN) substrate baseplate, copper plate below the substrate, and copper plates with a trench at the top. The copper is bonded to the ceramic substrate by direct copper bonding (DCB) method without any additional interlayers. ${ }^{3}$ The trench is created by etching. The etching creates sharp ends at the metallisation edges. The IGBT switch and chip diodes are located on top of the top-left copper plates. The diode consists of an active junction and ring termination as shown in the upper part of Figure 1. The termination consists of some diffused p-type semiconductor wells in n-substrate contacted by metal islands. These islands are interconnected by a semi-insulating layer. The active junction is metalised and the top of the active junction and ring termination is covered with a thin layer of polyimide. Opening the switch will create a potential difference across the trench. This creates field stress at chip edges and the trench between metallisation at the top of the ceramic substrate.

Unlike the usual practice of avoiding sharp edges in HV devices and components, sharp edges cannot be avoided due to the miniature nature of the power electronic components. Field enhancement occurs around these termination rings (field grading rings) on opening the switch. This field stress on conducting microfibre contamination can bridge two of the guard rings. This can trigger a complete system breakdown. To avoid this impact of conducting impurity, the surface of the module is covered with a thin polymer layer and the entire module is encapsulated in a soft dielectric such as silicone gel. 


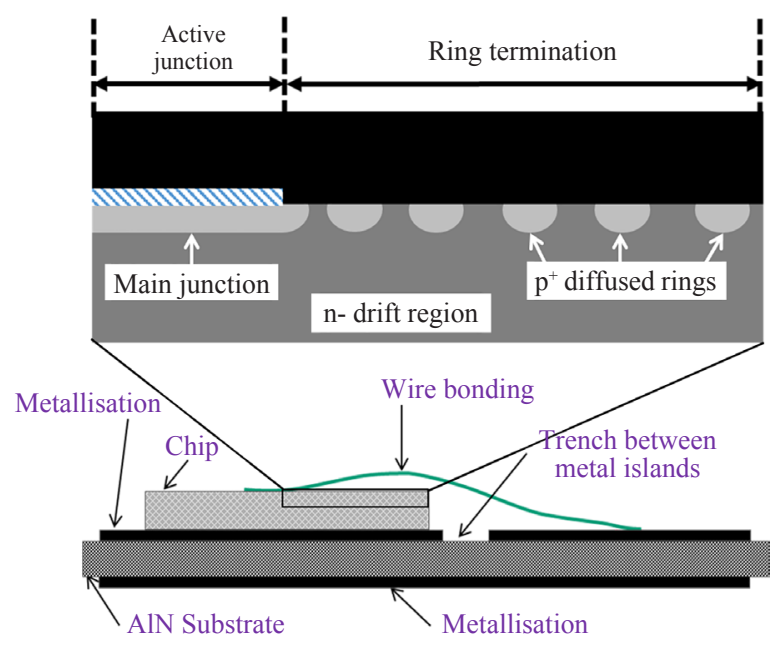

Figure 1: Schematic diagram of the IGBT module with the detail of active junction and guard ring termination.

The softness, good adhesive properties and the electrical insulating properties of silicone gel make it a good choice to isolate the circuits from the effects of impurities and avoid fractures from thermomechanical stresses. The gel has a high breakdown voltage and serves to prevent PDs that may occur at high voltages. ${ }^{4}$ It suppressed PDs produced at the sharp edges and increase the breakdown strength of the module. ${ }^{5}$ But a recent study on the degradation process of siliconegel by internal surface discharges calls for optimisation of insulation design for power electronics. ${ }^{6}$ Silicone gel as a semi-solid material is stagnant and initiation of streamers leads to the development of a cavity in the gel. This cavity expands every half cycle. This causes irreversible degradation of the gel. ${ }^{7}$ The presence of moisture in the module can lead to processes such as electrical treeing that can cause failure of the device. ${ }^{8,9}$ Changing the encapsulation of the power electronic module to an insulating material with self-healing properties is considered to be more desirable. In a quest to develop pressure tolerant power electronic for subsea application, insulating liquids are considered as a possible alternative encapsulating material for the IGBTs. These will also serve as a good heat transport medium and prevent electrical discharges in the module. ${ }^{7}$

There is also high electric field stress at metallisation along the trench of the IGBT baseplate. These resulted from unipolar switching. This can lead to PDs inception in the triple region. Our previous work on this topic was connected to short-term and long-term experiments for two critical high field areas, namely substrate trenches and chip edges, and we wanted to find new feasible material candidates and material combinations, i.e., liquid/solid insulation. ${ }^{10,11}$ 
The short-term experiment examined the integrity of some liquid samples (Nytro 10XN, Midel 7131 and Galden HT220) as an encapsulation material for the IGBT module and it was concluded that any of the liquids could be used for encapsulation but Galden has a better performance. ${ }^{10}$ In non-stagnant liquid insulation, impurities can be dragged towards the high field triple-point region by dielectrophoretic forces. Particles may lead to further enhancement of the field initiating PDs. However, the trench can be coated with a thin polymer film to keep away any impurities from the high field region. ${ }^{10}$

In the long-term endurance test of the component, uncoated chips were subjected to $6.5 \mathrm{kV}$ DC stress in an oil environment. The test object was observed to have a very low performance. Microscopic investigation of the failed objects revealed contaminations (fibres and other particles) at the surface and around the high field regions. The effect of these contaminants on the field distribution may probably be responsible for the failure of the components, thereby reducing the reliability of the proposed liquid encapsulation system. The failure of the chip diode in oil is an indication that the thin layer of polyimide covering the metallised active junction of the diode is not enough to shield the component from field enhancement due to the presence of impurities. Meanwhile, there was a report that the application of functional materials on a highly stressed region is capable of reducing the effect of electric field. ${ }^{12}$

The long-term experiment suggests that the presence of impurities in the proposed self-healing encapsulating liquid material may not make the materials to provide a complete reliable packaging system for the power electronic module since such impurity can be dragged to the high field region through the dielectrophoretic process. The numerical simulations, in this work, is to understand the failure risk of coated and uncoated IGBT related test object subjected to DC stress using finite element method (FEM) and then evaluate the operational reliability of chip diodes coated with polymer layer under varying relative humidity in comparison with other encapsulation materials.

\section{EXPERIMENTAL}

\subsection{Materials}

Dark brown freewheeling diode chips provided by Westcode were used as objects. The diodes were glued to a copper plate (in operation at HV) using silver epoxy (Epotek TV1003). A contact was glued to the centre of the diode for leakage current measurements. The diodes were cleaned using nap free clothing and thereafter inspected using a microscope and put into a zip bag. The component was then 
coated with parylene of thickness $27 \mu \mathrm{m}$ in a clean environment and thereafter again inspected in a microscope. ${ }^{11}$

\subsection{Field Distribution Simulation}

The breakdown voltage of the IGBT module is a key characteristic. It is necessary to have an edge termination structure that will reduce the electric field crowding at the diode and IGBT edges. ${ }^{13,14}$ A termination design based on the biased ring concept was employed in the design of the HV diode/IGBT chips. A diode was viewed under a microscope and the width of the guard rings and the gaps between the rings served as a guide in the simulation of the diodes guard rings and edge termination. The nine field limiting rings in the diode served as the basis for the chip simulation. The width of the guard ring was made to be $25 \mu \mathrm{m}$ as observed from the microscope. The gap increased towards channel stopper, a heavily doped n-type diffused region along the outer periphery. To design a system with breakdown voltage having a higher field ring, the depth of junction should be as deep as possible. ${ }^{14}$ The depth of the p-well (junction) in the diode was assumed to be $20 \mu \mathrm{m}$. Figure 2 shows the meshed field limiting ring (also shown schematically in Figure 1) that divides the entire space into a small rectangular size for calculation using the FEM.

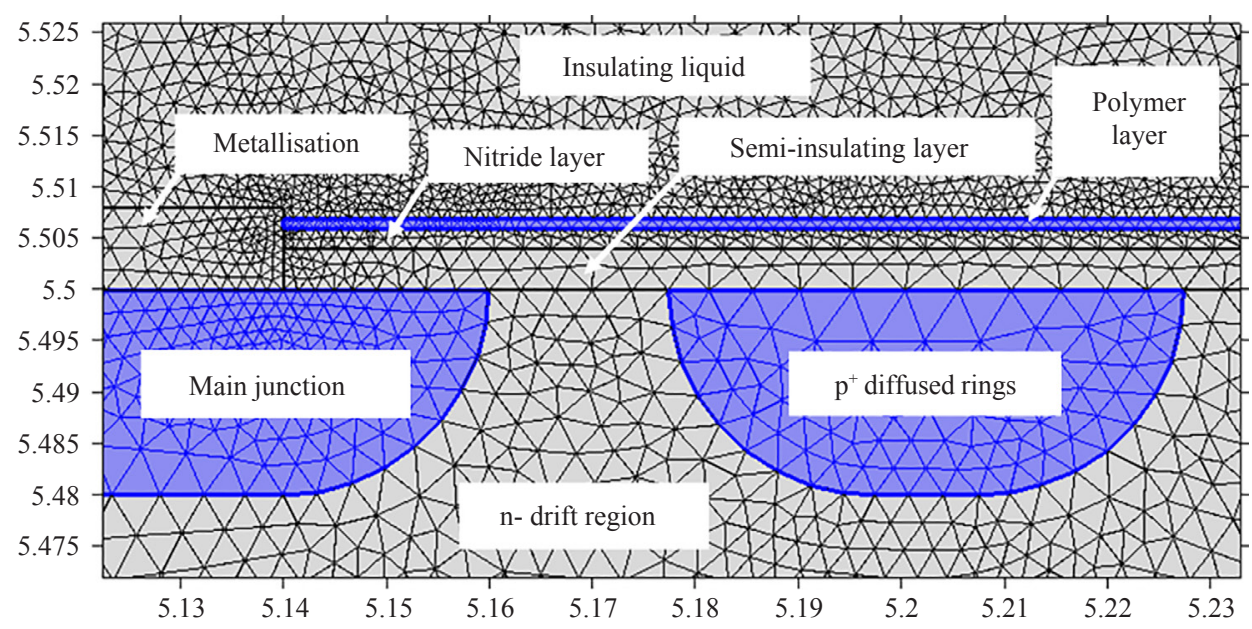

Figure 2: Chip diode termination rings covered with thin polymer layer (numbers on the axis are the position in $\mathrm{mm}$ ). 
The calculation was done to solve the Poisson difference equations everywhere in the device using a charge density equal to the net impurity charge. The voltage on each of the field limiting ring was estimated from the Poisson's equation:

$$
\frac{1}{r} \frac{\partial}{\partial r}\left(r \frac{d V}{d r}\right)=-\frac{q N_{d}}{\varepsilon_{s i}} \quad r_{j} \leq r \leq r_{d}
$$

where $N_{d}$ is the doping density of the epitaxial layer which was taken as $1 \times 10^{14} \mathrm{~cm}^{-3}, \varepsilon_{s i}$ is the permittivity of silicon, $r_{j}$ is the depth of diffused ring, $r_{d}$ is the radius of the depletion region in the epitaxial layer which in this simulation is assumed to be equal to the ring separation, $V$ is the ring junction voltage, and $q$ is an electronic charge. ${ }^{15-17}$ The metallisation on top of the ring was interconnected with semi-insulating polycrystalline silicon (SIPOS) of thickness $4 \mu \mathrm{m}$. The SIPOS and metallisation were then covered with a nitride layer of thickness $2 \mu \mathrm{m}$. On top of the nitride was a layer of polymer (polyimide) of thickness $1 \mu \mathrm{m}$. Table 1 shows the floating voltage for the field limiting rings. Field limiting ring 1 is the ring next to the active junction. An amount of $4.5 \mathrm{kV}$ semi-square voltage with fast rise-time was simulated and applied to the back contact. The active junction was grounded and the field limiting rings were left floating.

Table 1: Voltage distribution across the chip termination rings.

\begin{tabular}{ccc}
\hline Field limiting rings & Inter ring distance $(\mu \mathrm{m})$ & Voltage $(\mathrm{V})$ \\
\hline 1 & 17.5 & 0.5 \\
2 & 30.5 & 10.0 \\
3 & 40.0 & 39.0 \\
4 & 50.0 & 95.0 \\
5 & 60.0 & 181.0 \\
6 & 80.0 & 450.0 \\
7 & 95.0 & 747.0 \\
8 & 120.0 & $1,440.0$ \\
9 & 150.0 & $2,630.0$ \\
\hline
\end{tabular}

\subsection{Experimental Setup}

The surface of the diode was cleaned and a parylene film of thickness $27 \mu \mathrm{m}$ was applied on the surface by condensing a polymer vapour onto the object. Parylene is a thermoplastic polymer that is based on para-xylylene and applied using vapour phase polymerisation. The choice of parylene as the coating polymer was because parylene was reported to be a completely conformal coating, of uniform thickness and pinhole-free for the protection of devices, components and surfaces in electronics. ${ }^{18}$ It has a breakdown strength of about $10 \mathrm{MV} \mathrm{cm}{ }^{-1} .{ }^{19}$ Parylene 
coated chip diodes were then put under DC voltage stress while embedded in the insulation liquid. This was done at high and low humidity at ambient pressure. ${ }^{9}$ Other diode chips were similarly cleaned and coated with silicone gel of thickness $1 \mathrm{~cm}$ and embedded in insulation liquid and subjected to voltage stress.

Figure 3 shows the setup for the long-term experiment on the functionality of electrically stressed components. The leakage current was continuously monitored for each object and the component was considered failed when the current exceeded a certain level. A drop in the voltage due to the limitation of the total current delivered by the voltage source also indicates failure. The humidity control was taken care of using filtered air (to avoid particles) flow bubbling through a water reservoir at the incubator temperature. The humidified air was then guided into the humidity control chamber (sealed polymer vessels) containing the objects in operation and mixed with dry air at the appropriate rate to achieve dry/wet humidity environments.

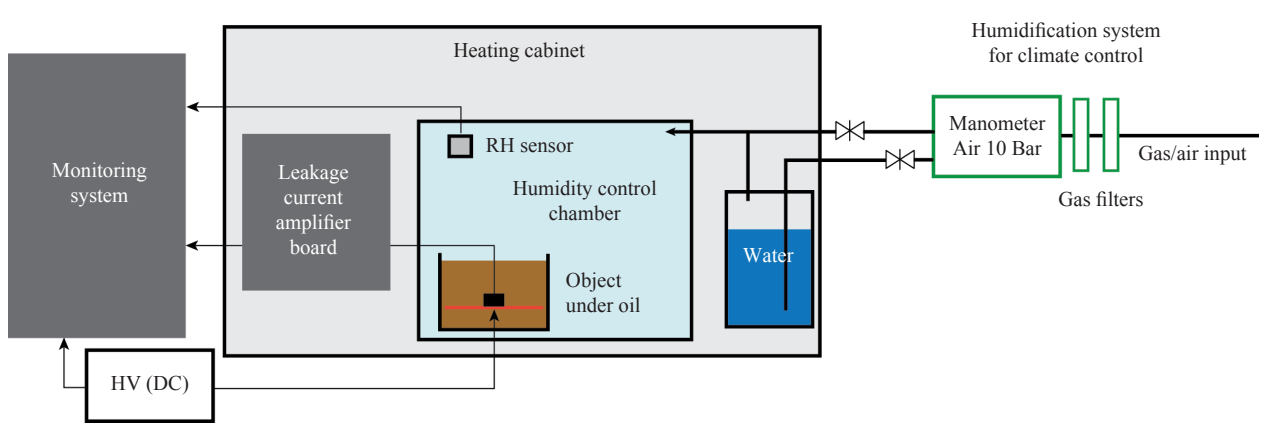

Figure 3: Schematic of the experimental setup for the long-term ageing experiment.

\section{RESULTS AND DISCUSSIONS}

The numerical analysis of the field around the biased rings shows that the field limiting rings spread the voltage across the ring termination as shown in Figure 4 which displayed the electric potential in $\mathrm{kV}$. The contour lines in the figure show the potential distribution in $\mathrm{kV}$. Ring 4 has a contour line with an electric potential of $0.13 \mathrm{kV}$ around it. The third contour line after rings 7 and 8 have a potential of $1.4 \mathrm{kV}$ and $1.6 \mathrm{kV}$, respectively. And the fourth contour line after ring 9 has a potential of $3.4 \mathrm{kV}$. The breakdown in the IGBT module usually occurs at the point of maximum curvature. The field ring helps reduce the effect of junction curvature as its introduction caused the lines of constant potential not to follow the shape of the curved junction. ${ }^{20}$ It eliminates the crowding of high fields and current at the anode periphery during reverse recovery. The field grading designs 
provide less termination area to utilise a more active region for conduction. The lines of constant potential assume a shape with less curvature, and thus, reducing the peak value of the field. The point of the maximum electric field is shifted from the surface to the substrate interior to improve the breakdown characteristic and the reliability of the chip. ${ }^{21,22}$ Depending on the thickness of the insulating layer covering the surface of the diode, a conducting fibre on the surface can lead to field enhancement that can be high enough to initiate a pre-breakdown phenomenon on the IGBT module. The observed fibre on the diode chip from the earlier report was simulated. ${ }^{9}$

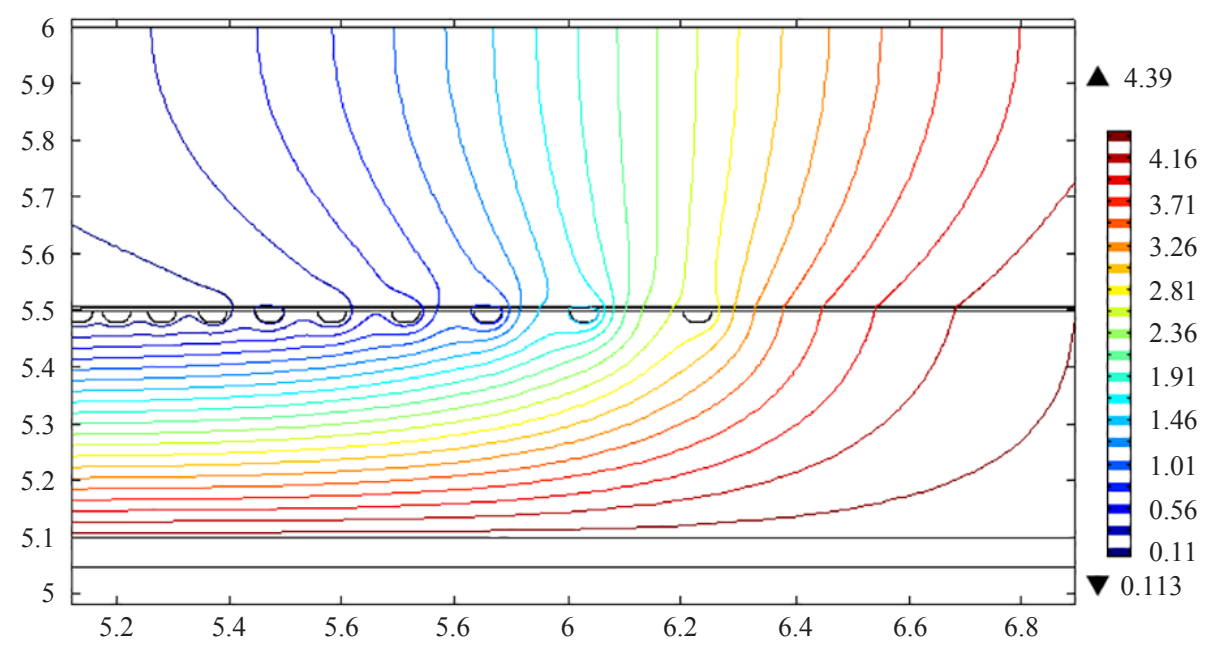

Figure 4: Electric potential in $\mathrm{kV}$ across the termination area (the numbers on the axis are the position in $\mathrm{mm}$ ).

Figure 5 shows the electric field distribution in $\mathrm{kV} \mathrm{m}^{-1}$ around the guard rings (7, 8 and 9). The blue areas indicate the region with the maximum electric field which is the most important parameter in the design of the field limiting ring. The edge peak electric field for the main junction and junction rings is shown in Figure 6 . The edge peak electric field of the main junction for a no-field limiting ring device is responsible for the breakdown. The introduction of multiple fields limiting rings to the device is to reduce the edge peak electric field of the main junction. This is due to the transfer of the voltage from the main junction to the first guard ring junction. The second field limiting ring does the same to the voltage and edge peak field of the first ring junction. This process continues through to the last ring. ${ }^{20}$ The reduction in the edge peak field brings about an increase in the breakdown field of the device. 


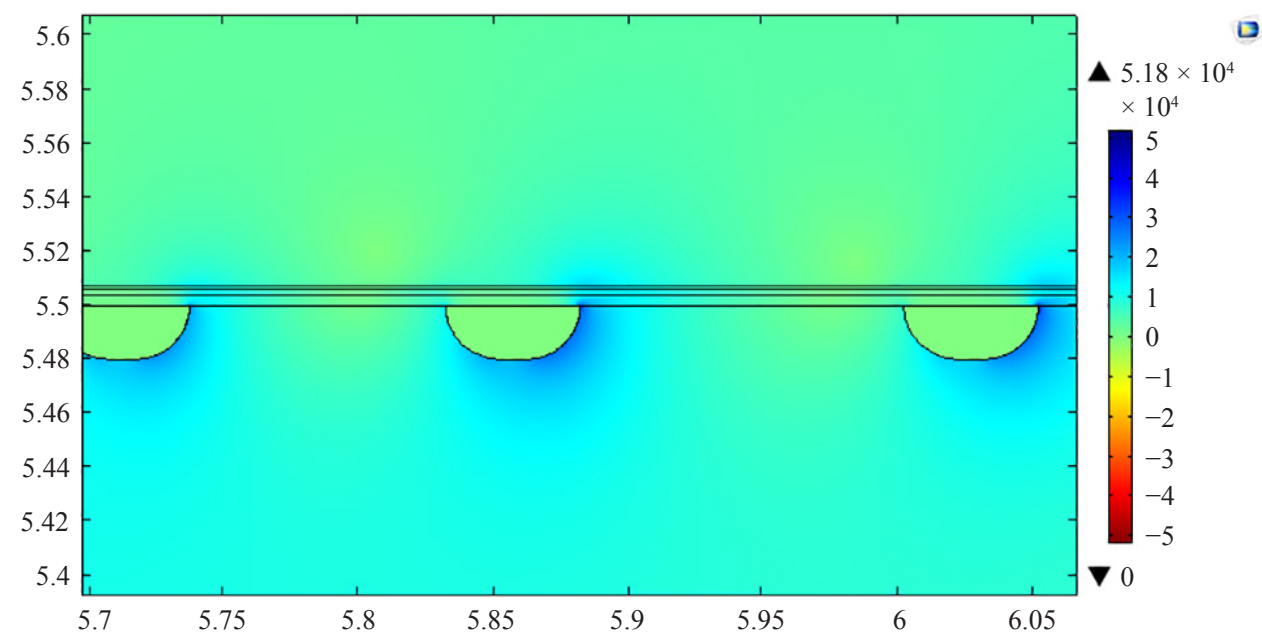

Figure 5: Electric field distribution $\left(\mathrm{kV} \mathrm{m}^{-1}\right)$ with regions of field peaks on the multiplefield limiting rings (numbers on the axis are the position in $\mathrm{mm}$ ).

However, this edge peak field can cause field enhancement to the field distribution around a conducting fibre impurity at the surface of a chip diode. This can result in the breakdown of the device. Increasing the polymer layer at the top of the diode may reduce this effect of this field enhancement. Therefore, a two-dimensional conducting fibre impurity of rectangular shape with a length of $600 \mu \mathrm{m}$ and thickness of $0.11 \mu \mathrm{m}$ was simulated on top of the polymer layer on the diode ring termination. The thickness of the polymer layer was varied from $1 \mu \mathrm{m}$ to $35 \mu \mathrm{m}$ to examine the influence of the thickness on the field enhancement of a conducting impurity on top of the polymer. The geometry of the fibre was kept constant when increasing the polyimide layer thickness. With a thickness of $1 \mu \mathrm{m}$, a maximum field enhancement of $3.69 \times 10^{4} \mathrm{kV} \mathrm{m}^{-1}$ was observed. This field enhancement could be high enough to cause the breakdown of the IGBT module. This may have resulted in the breakdown of the surface of the chip as reported. ${ }^{11}$

Increasing the thickness of the polymer layer reduced the field enhancement on the fibre impurity as shown in Figure 7. As the thickness of the insulating polymer increased, a decrease in field enhancement was observed. This effect is more pronounced up to the thickness of $20 \mu \mathrm{m}$. The maximum field in the polymer was evaluated to be about $1.88 \times 10^{4} \mathrm{kV} \mathrm{m}^{-1}$ from Figure 5 . This is low compared with the breakdown field of polyimide film reported being above $2 \mathrm{MV} \mathrm{cm} \mathrm{cm}^{-1}$ $\left(2 \times 10^{5} \mathrm{kV} \mathrm{m}^{-1}\right)$, an indication that local breakdown is not expected from the polymer in the absence of voids. ${ }^{21}$ 


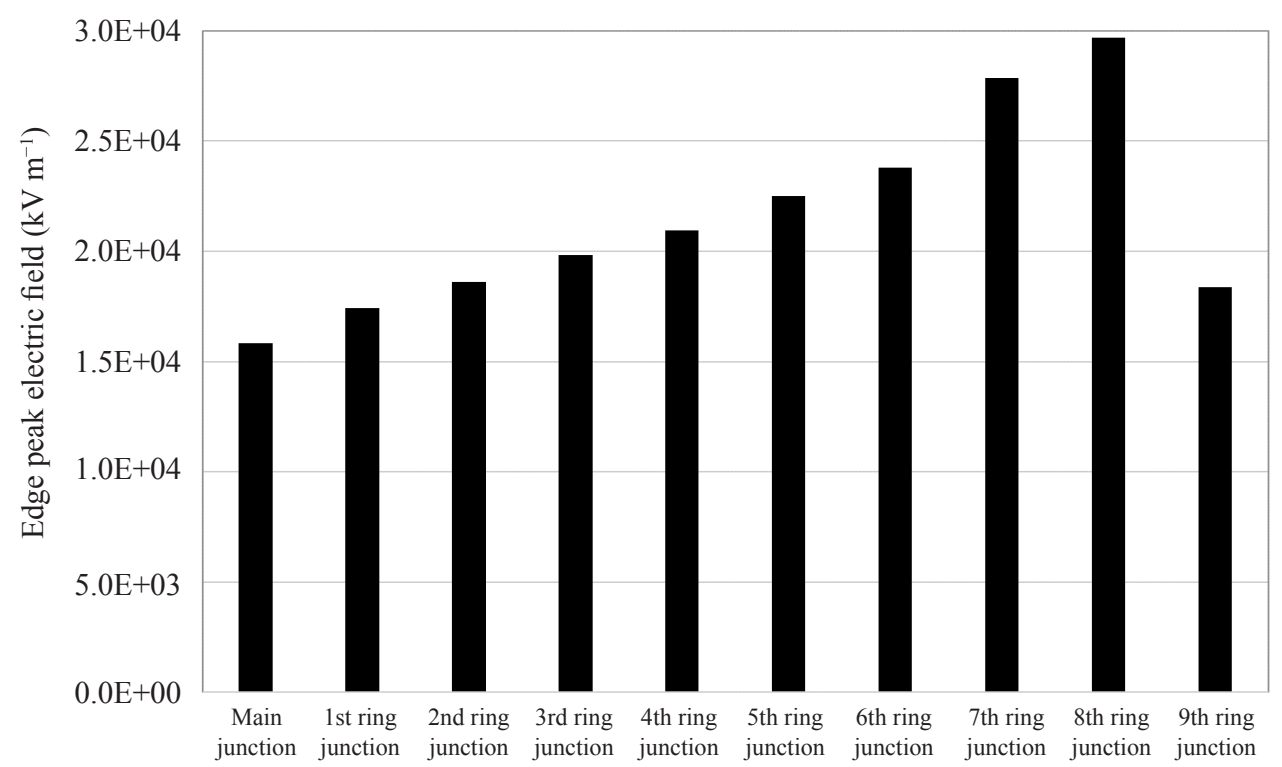

Figure 6: The plot of the edge peak electric field of the simulated main junction and the 9 field limiting ring junctions under $4.5 \mathrm{kV}$ semi-square voltage.

The difference between the simulations and the long-term experiment is that for the simulation, a fast rise square voltage was applied while for the long-term test, it utilised a constant voltage that remained stable from turn-on till the object failed. The simulation focus on the high field phenomena during switching while the long-term experiment operates at constant DC voltage (except for ripple effects) for a long time.

Parylene-coated chips placed in a liquid environment (Midel 7131) and subjected to $6.5 \mathrm{kV}$ DC stress as described in the setup in Figure 3 examined the impact of the enhanced coating polymer layer on the performance and reliability of IGBT module. The polymer-coated components (dry environment of $3 \%$ relative humidity $(\mathrm{RH})$ ) were observed to have increased the operation time under service condition as seen in Figure 8 when compared with the earlier result obtained from the uncoated components. ${ }^{11}$ The coated components were in operation at service condition for 67 weeks under experimental conditions without failure of the objects under test. This is an indication that the application of an additional thin polymer coat on the test objects was able to shield the impurities that could have been dragged towards the high field region of the active junction, causing field enhancement and eventual breakdown as it occurred for the uncoated components. Increasing the thickness of the polymer layer for the active region of the chip will protect the components better both during switching and when operating at a constant DC voltage. 


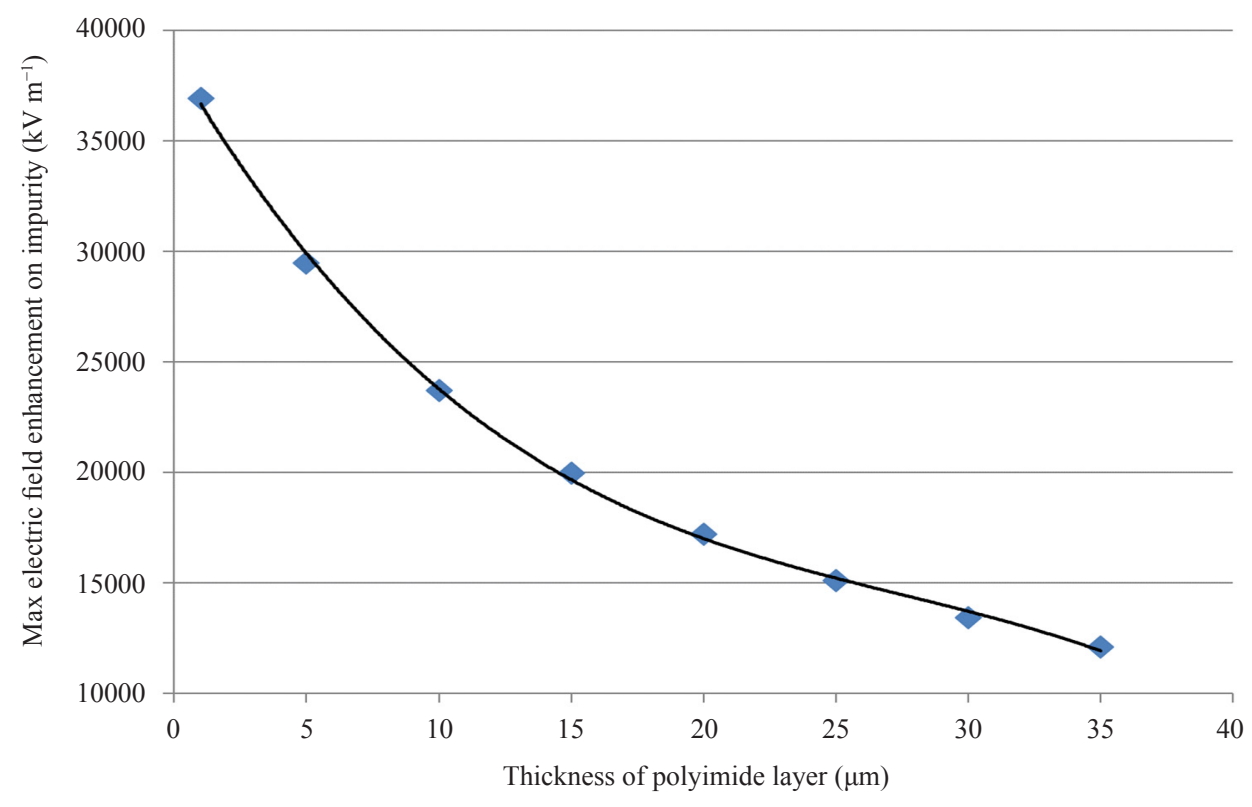

Figure 7: Field enhancement on impurity versus polymer thickness.

The effect of wet humidity environments was investigated on the polymercoated objects in Midel. Figure 8 displays the effect of high RH $(70 \% \mathrm{RH})$ for the parylene coating. Rapid failure of the polymer-coated objects embedded in Midel (wet) was observed as compared with the dry objects that remain in operation within the operation time. The poor performance of the polymer-coated chip in a Midel under $70 \%$ RH may be due to the initiation of water treeing. The presence of high humidity may have initiated the growth of a water tree at any protrusion with high stress. Another breakdown mechanism may have taken over to govern the growth of trees to failure. A test was then conducted on test objects but coated with thick silicone gel and embedded in insulation liquids; FR3 Envirotemp and Galden HT220, respectively. The silicone gel coated objects in insulation oil were observed to remain in operation at service condition and $3 \% \mathrm{RH}$ after 67 weeks when the experiment ended without failure as shown in Figure 9 . RH of $70 \%$ shows a decrease in the service life of the silicone gel coated chips in Figure 9. Meanwhile, a comparison between Figures 8 and 9 shows that in a wet environment (environment with high $\mathrm{RH}$ ), the thick silicone gel coat protects the chips longer than the thin polymer coating. The decrease in the service life with increased RH is still an indication that humidity penetrates the thick coating with time, leading to a failure caused by water tree growth while the dry objects remain in operation. ${ }^{20}$ 
Environment and material combinations

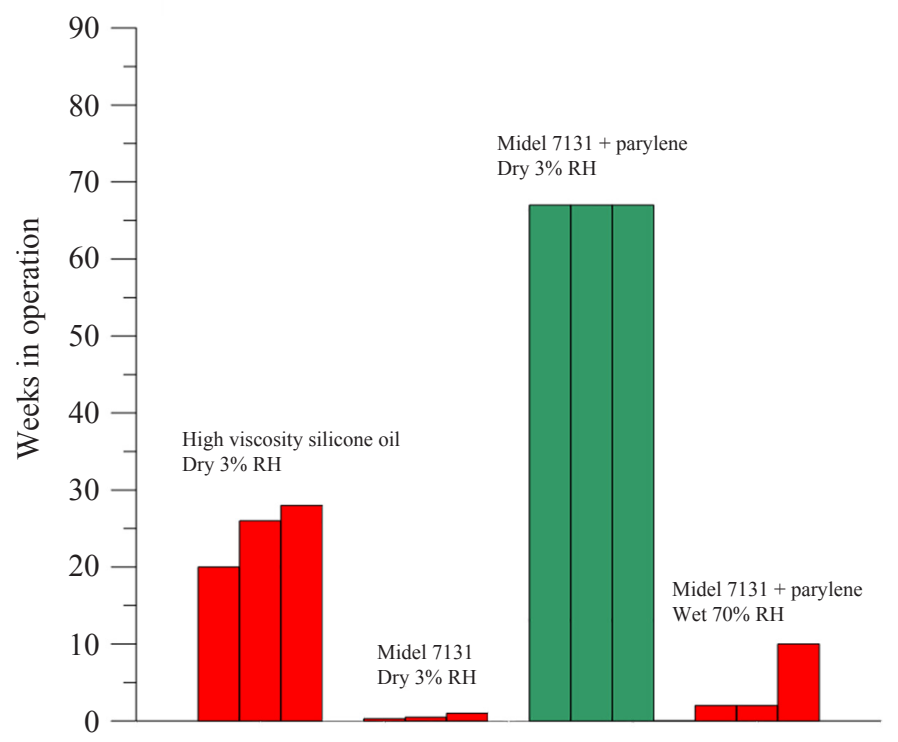

Figure 8: Chip objects in operation in oil for "no coated" and thin (parylene) coated objects at dry and wet conditions.

Environment and material combinations

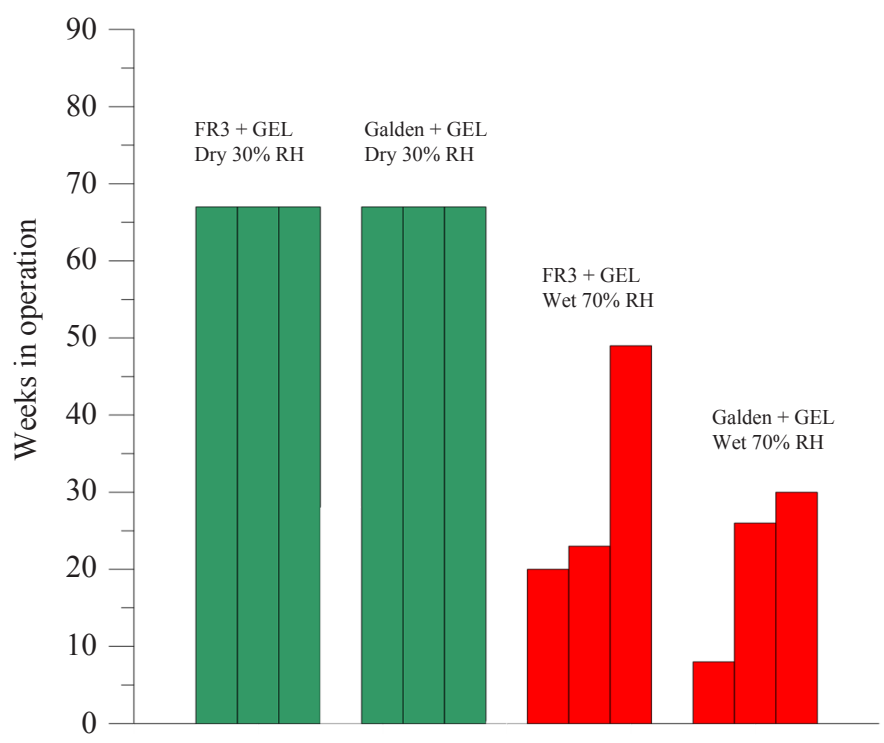

Figure 9: Chip objects in operation in oil for silicone gel coated objects $(1 \mathrm{~cm})$ at dry and wet conditions. 


\section{CONCLUSION}

The FEM calculation suggests that increasing the thickness of the polymer insulation layer on the guard rings and edge termination will significantly reduce field enhancement on impurities on the surface of the diode thereby reducing the probability of breakdown on the surface of the diode. Polymer layer of a thickness of $20 \mu \mathrm{m}$ or more is suggested to be sufficient to protect the chip from early breakdown. The application of $6.5 \mathrm{kV}$ DC stress on the uncoated components in the oil environment resulted in the failure of the component after less than one week which may have been due to fibres at the surface and around the high field regions. The long-term experiment at medium elevated oil temperature and dry service conditions covered with insulation liquid revealed that test objects with the additional coated polymer layer of thickness $27 \mu \mathrm{m}$ did not experience failure within the study time of 67 weeks under dry operation condition.

High humidity, however, provokes the failure of polymer-coated chips in oil within a few weeks in operation. The chips with a thicker coating such as silicone gel produced results similar to parylene coated chips under the dry condition and will protect the object longer compare to a thinner coating under higher relative humidity.

Since impurities can hardly be avoided, increasing the thin polymer coat on the active region of the IGBT module to a thickness of about $27 \mu \mathrm{m}$ and embedded in an insulating liquid environment may be a better encapsulation system for power electronic module under dry condition. Thick silicone gels as encapsulation will withstand a humid environment longer but moisture will still penetrate in the end to shorten the life span.

\section{ACKNOWLEDGEMENTS}

The authors wish to acknowledge the contributions of Lars E. Lundgaard at SINTEF Energy Research.

\section{REFERENCES}

1. Kopta, A. et al. (2008). 6500V SPT + HiPak modules rated at 750A. Paper presented at the Proceedings of International Exhibition and Conference for Power Electronics, Intelligence Motion, Renewable Energy and Energy Management, Nuremberg, 27-29 May, 1-7. 
2. Corvasce, C. et al. (2011). New 1700V SPT+ IGBT and diode chip set with $175^{\circ} \mathrm{C}$ operating junction temperature. Paper presented at the Proceedings of the 2011 14th European Conference on Power Electronics \& Application, Birmingham, 30 August-1 September, 1-10.

3. Schulz-Harder, J. (2003). Advantages and new development of direct bonded copper substrates. Microelectr. Reliab., 43(3), 359-365. https://doi.org/10.1016/ S0026-2714(02)00343-8

4. Do, M. T., Augé, J.-L. \& Lesaint, O. (2006). Dielectric losses and breakdown in silicone gel. Annual Report on IEEE Conference on Electrical Insulation and Dielectric Phenomena, 541-544. https://doi.org/10.1109/CEIDP.2006.311989

5. Augé, J.-L., Lesaint O. \& Vu Thi, A. T. (2003). Partial discharges in ceramic substrates embedded in liquids and gels. IEEE Trans. Dielect. Elect. Ins., 20(1), 262-274. https://doi.org/10.1109/TDEI.2013.6451366

6. Locatelli, M. et al. (2014). Evaluation of encapsulation materials for hightemperature power device packaging. IEEE Trans. Pow. Electr., 29(5), 2281-2288. https://doi.org/10.1109/TPEL.2013.2279997

7. Sato, M., Kumada, A. \& Hidaka, K. (2014). On the nature of surface discharges in silicone-gel: Prebreakdown discharges in cavities. Paper presented at the Proceedings of the IEEE Conference on Electrical Insulation and Dielectric Phenomena, Des Moines, 19-22 October. https://doi.org/10.1109/ CEIDP.2014.6995729

8. Papadopoulos, C. et al. (2018). The influence of humidity on the high voltage blocking reliability of power IGBT modules and means of protection. Microelectr. Reliab., 88-90, 470-475.

9. Papadopoulos, C. et al. (2019). Humidity robustness of IGBT guard ring termination. Paper presented at the International Exhibition and Conference for Power Electronics, Intelligent Motion, Renewable Energy and Energy Management, Nuremberg, Germany, 1-8.

10. Abdelmalik, A. A., Nysveen, A. \& Lundgaard, L. E. (2016). Partial discharges in liquid embedded power electronics: Effects of pressure and liquid nature under negative pulse voltage stress. IEEE Trans. Dielect. Elect. Insul., 23(2), 1119-1125. https://doi.org/10.1109/TDEI.2015.005615

11. Liland, K. B. et al. (2016). Liquid insulation of IGBT modules: Long term chemical compatibility, and high voltage endurance testing. Paper presented at the Proceedings of the IEEE International Conference on Dielectrics, Montpellier, 3-7 July. https://doi.org/10.1109/ICD.2016.7547624

12. Ghassemi, M. (2018). Electrical insulation weaknesses in wide bandgap devices. In Sanchez, R. A. (Ed.), Simulation and modelling of electrical insulation weaknesses in electrical equipment. London: IntechOpen, 129-149. https://doi. org/10.5772/intechopen.77657

13. Saxena, V., Su, J. N. \& Steckl, A. J. (1999). High-voltage Ni- and Pt-SiC Schottky diodes utilizing metal field plate termination. IEEE Trans. Electr. Dev., 46(3), 456-464. https://doi.org/10.1109/16.748862 
14. Jung, E. S. et al. (2014). A study of field-ring design using a variety of analysis method in insulated gate bipolar transistor (IGBT). J. Electr. Eng. Technol., 9(6), 1995-2003. http://dx.doi.org/10.5370/JEET.2014.9.6.1995

15. Adler, M. S., Temple, V. A. K. \& Ferro, A. P. (1975). Breakdown voltage for planar devices with a single field limiting ring. Paper presented at the IEEE Power Electronic Specialists Conference, 300-304.

16. Adler, M. S. et al. (1977). Theory and breakdown voltage for planar devices with a single field limiting ring. IEEE Trans. Electr. Dev., 24(2), 107-112. https://doi.org/10.1109/T-ED.1977.18688

17. Kim, Y. H. et al. (2008). A new edge termination technique to improve voltage blocking capability and reliability of field limiting ring for power devices. Paper presented at the Proceedings of the IEEE International Conference on Integrated Circuit Design \& Technology \& Tutorial, Texas, 2-4 June, 71-74. https://doi.org/10.1109/ICICDT.2008.4567249

18. Morris, J. R. (1995). Electrosurgical instrument having a parylene coating. Patent US5380320 A.

19. Diaham, S. et al. (2014). Dielectric strength of parylene HT. J. Appl. Phys., 115(5), 054102. https://doi.org/10.1063/1.4863877

20. He, J. et al. (2006). A new analytic method to design multiple floating fields limiting rings of power devices. Sol-State Electr., 50(7-8), 1375-1381. https://doi.org/10.1016/j.sse.2006.06.012

21. Diaham, S. et al. (2010). Dielectric breakdown of polyimide films: Area, thickness and temperature dependence. IEEE Trans. Dielect. Elect. Insul., 17(1), 18-27. https://doi.org/10.1109/TDEI.2010.5411997

22. Nunes, S. L. \& Shaw, M. T. (1980). Water treeing in polyethylene - A review of mechanisms. IEEE Trans. Dielect. Elect. Insul., 17(1), 437-450. https://doi.org/10.1109/TEI.1980.298272 